\title{
O Papel dos Preços e do Dispêndio no Consumo de Alimentos Orgânicos e Convencionais no Brasil ${ }^{1}$
}

\author{
Alberes Sousa Ferreira² e Alexandre Bragança Coelho ${ }^{3}$
}

Resumo: Este artigo buscou analisar a sensibilidade da demanda de alimentos orgânicos e suas contrapartes convencionais frente a variações nos preços e no dispêndio nos domicílios brasileiros. Para isso, estimou-se um sistema de demanda para 14 categorias agregadas (sete orgânicas e sete convencionais) por meio do modelo QUAIDS com a correção do Problema do Consumo Zero pelo procedimento de Shonkwiller e Yen, utilizando-se os microdados extraídos da Pesquisa de Orçamentos Familiares (POF) 20082009. Constatou-se que os consumidores brasileiros são mais sensíveis a variações nos preços e dispêndio dos orgânicos do que dos convencionais. Pôde-se confirmar também que há assimetria nas relações de substituição/complementaridade entre os dois tipos de alimentos. Assim, é relativamente difícil induzir consumidores habituados a adquirir produtos orgânicos a "reverterem" seus hábitos de consumo, trocando produtos orgânicos por convencionais. Além disso, pôde-se notar que os alimentos orgânicos ainda não são vistos pelos consumidores como substitutos dos convencionais na maioria dos casos. Verificou-se, ainda, por meio das elasticidades-dispêndio, que os alimentos orgânicos podem ser classificados como bens de luxo no Brasil.

Palavras-chaves: alimentos orgânicos, demanda, modelo QUAIDS.

\begin{abstract}
This article seeks to analyze the demand's sensibility for organic food and their conventional counterparts to variations in prices and expenditure in Brazilian households. We estimated an aggregate demand system for 14 categories (seven organics and seven conventional) using the QUAIDS model with correction for the Zero Consumption Problem by the Shonkwiller and Yen twostep estimation method. The database was from the microdata from the Brazilian Household Budget Survey (POF/IBGE) 2008/2009. Results showed that Brazilian consumers are more sensitive to variations in prices and expenditure of organic than conventional food. Moreover, there is asymmetry in substitution/complementarity relations between the two types of food. These results suggest that it is relatively difficult to induce consumers that are used to purchase organic products to "revert" their spending habits changing organic products to conventional ones. Furthermore, results showed that
\end{abstract}

1. Data de submissão: 18 de dezembro de 2015. Data de aceite: 4 de julho de 2017.

2. Universidade Federal de Viçosa (UFV), Viçosa-MG, Brasil. E-mail: alberesasf@yahoo.com.br

3. Universidade Federal de Viçosa (UFV), Viçosa-MG, Brasil. Bolsista de Produtividade em Pesquisa do CNPq. O autor agradece o apoio financeiro do CNPq. E-mail: acoelho@ufv.br 
consumers do not view organic food as a substitute for conventional food in most cases. Examining the expenditure elasticities, we conclude that organic foods can be classified as luxury goods.

Key-words: organic food, demand, QUAIDS model.

Classificação JEL: C34, D12, R21.

DOI: http://dx.doi.org/10.1590/1234-56781806-94790550401

\section{Introdução}

Embora a prática da agricultura seja exercida pela humanidade há cerca de dez mil anos, apenas com o término da Segunda Guerra Mundial se verificou a implementação de novas técnicas produtivas que levaram ao grande aumento da produção agrícola. Estas novas técnicas de produção baseavam-se na intensificação da utilização de produtos químicos, desenvolvimento de pesquisas em sementes, fertilização do solo e mecanização no campo. Este processo de fomento à ampliação da produtividade na agricultura ficou conhecido como "Revolução Verde" (MAZOYER e ROUDART, 2010). No entanto, essas práticas agrícolas fundamentadas na utilização maciça de produtos químicos têm gerado preocupações crescentes quanto aos riscos à saúde humana e ao meio ambiente. Desse modo, a busca por alimentos mais saudáveis, provenientes de sistemas de produção mais sustentáveis, como os métodos orgânicos de produção, é uma tendência que vem se fortalecendo e se consolidando em nível mundial (SOUZA, 2003).

O mercado brasileiro de alimentos orgânicos segue essa tendência mundial. Segundo informações divulgadas pelo Projeto Organics Brasil, as vendas de produtos orgânicos no Brasil atingiram a marca de R \$ 350 milhões em 2010, valor 40\% superior ao registrado no ano anterior (MAPA, 2014). Ainda de acordo com informações do programa, as exportações aumentaram de cerca U\$ 9,5 milhões, em 2005, para aproximadamente U\$ 130 milhões em 2013 (ORGANICS BRASIL,
2014). Quanto aos produtores agrícolas que trabalham segundo as normas dos sistemas orgânicos de produção nacional, verificou-se aumento de 51,7\% entre 2014 e 2015. Foram registrados 6.719 produtores em janeiro de 2014, e 10.194 em janeiro de 2015. As Unidades de Produção de orgânicos também apresentaram significativo aumento: passaram de 10.064 unidades em janeiro de 2014 para 13.323 no mesmo mês de 2015, acréscimo de 32\% (MAPA, 2015). Além disso, a taxa de crescimento do setor no País apresenta índices que variam de $30 \%$ a $40 \%$ ao ano (PORTAL ORGÂNICO, 2015); enquanto taxas de crescimento de até $10 \%$ a.a. foram verificadas nos mercados de orgânicos mais desenvolvidos até 2012 (WILLER e LERNOUD, 2014).

Por mais que se reconheça a importância que o mercado de orgânicos vem assumindo ao longo dos últimos anos, ainda pouco se sabe sobre os principais fatores que influenciam a demanda por este segmento no Brasil e sobre a relação existente entre a demanda por alimentos orgânicos e convencionais 4 . Investigar a influência dos preços e da renda, bem como as relações de substitutibilidade e complementaridade entre ali-

4. Apesar de a demanda domiciliar por alimentos orgânicos poder ser influenciada pelos preços de outros alimentos que integram a cesta de consumo dos domicílios, Fourmouzi, Genius e Midmore (2012) advertem que grande parte dos estudos de demanda sobre orgânicos negligenciam a presença de produtos rivais no mercado. Desse modo, no presente estudo incorporam-se - além dos alimentos orgânicos - suas contrapartes convencionais com características semelhantes. Por exemplo: frutas orgânicas e frutas convencionais. 
mentos orgânicos e convencionais, é de fundamental importância para a compreensão do comportamento dos consumidores deste mercado tão promissor.

Embora existam algumas pesquisas que busquem traçar o perfil do consumidor de alimentos orgânicos no Brasil (ASSIS, AREZZO e DE-POLLI, 1995; CERVEIRA e CASTRO, 1999; ARCHANJO, BRITO e SAUERBECK, 2001), ainda há poucas evidências sobre a sensibilidade do consumidor brasileiro de alimentos orgânicos em relação aos preços e ao dispêndio. Para o Brasil, apenas Oliveira e Hoffmann (2015) buscaram estimar a elasticidade-renda para orgânicos utilizando os microdados da POF; entretanto, não utilizam um sistema de demanda como neste trabalho. Além disso, desconhece-se também a magnitude e o sentido da substituição entre alimentos orgânicos e convencionais, mensurados pela elasticidade-preço cruzada entre os bens. Apesar de existirem estudos nacionais que indiquem que os consumidores de orgânicos apresentam maiores níveis de renda e de educação (CERVEIRA e CASTRO, 1999; ARCHANJO, BRITO e SAUERBECK, 2001), além de fazerem inferências sobre a percepção dos consumidores com relação às propriedades dos alimentos orgânicos, nenhum deles estimou um sistema de demanda para testar estas hipóteses.

Internacionalmente, diversos estudos buscaram investigar a demanda por orgânicos, especialmente nos Estados Unidos da América (EUA) (TREGEAR, DENT e MCGREGOR, 1994; WANG e SUN, 2003; LI, ZEPEDA e GOULD, 2007; GRACIA e MAGISTRIS, 2008; SMITH, HUANG e LIN, 2009; LIN et al., 2009; KASTERIDIS e YEN, 2012). Merecem destaque os trabalhos de Kasteridis e Yen (2012) e Lin et al. (2009), que buscaram investigar simultaneamente as demandas por alimentos orgânicos e seus correspondentes convencionais nos EUA. De acordo com o estudo de Lin et al. (2009), os consumidores norte-americanos são mais sensíveis a mudanças de preços das frutas orgânicas do que das frutas convencionais. Além disso, os efeitos-preço cruzados estimados sugerem que uma mudança nos preços relativos, com queda nos preços das frutas orgânicas, por exemplo, irão provavelmente induzir os consumidores a mudar de frutas convencionais para frutas orgânicas, enquanto é menos provável que o inverso aconteça quando há aumento nos preços das frutas orgânicas. Os demais pesquisadores que também buscaram compreender as inter-relações entre as demandas de alimentos orgânicos e conven- cionais (GLASER e THOMPSON, 1998; FOURMOUZI, GENIUS e MIDMORE, 2012) também encontraram relações semelhantes à do estudo supracitado. Se realmente houver maior sensibilidade da demanda de orgânicos em relação aos preços no Brasil, espera-se que uma queda nos mesmos amplie largamente o consumo de tais bens no País.

Este estudo tem, assim, o objetivo de investigar a sensibilidade dos consumidores brasileiros frente a variações nos preços e no dispêndio. Objetiva-se analisar também a relação de substituição/complementaridade entre alimentos orgânicos e convencionais, dada pelas elasticidades-preço cruzadas. Para alcançar este objetivo, este estudo utilizará os dados da Pesquisa de Orçamentos Familiares - POF 2008-2009, que pela primeira vez investigou com detalhes os hábitos de consumo de alimentos orgânicos no Brasil. Os resultados encontrados serão importantes por permitirem compreender a estrutura do consumo de alimentos orgânicos nos domicílios brasileiros. Estas informações são de grande utilidade para produtores e membros do setor varejista, que podem balizar de modo mais preciso suas decisões de precificação. Ademais, os resultados contidos neste estudo são de grande valia para o planejamento de políticas públicas que visem ampliar o consumo de alimentos orgânicos.

A próxima seção apresenta o referencial analítico deste trabalho. Na seção 3, apresentam-se a base de dados e as variáveis utilizadas. Nas seções 4 e 5 são apresentados os principais resultados e as considerações finais, respectivamente.

\section{Referencial analítico}

O Procedimento de Shonkwiller e Yen (1999) é um método de estimação em dois estágios que permite a inclusão de todas as observações da amostra. No primeiro estágio, estima-se um modelo de escolha binária, que permite determinar a probabilidade de aquisição dos bens em análise dentre os domicílios da amostra em função de características sociodemográficas. Já o segundo estágio considera a estimação do sistema de demanda. O procedimento pode ser apresentado como:

Primeiro estágio:

$$
d_{i k}^{*}=z_{i k}^{\prime} a_{i}+\vartheta_{i k},
$$


$d_{i k}=\left\{\begin{array}{l}1 \text { se } d_{i k}^{*}>0 \\ 0 \text { se } d_{i k}^{*} \leq 0\end{array}\right.$

$y_{i k}=d_{i k} y_{i k}^{*}(i=1, \ldots, m ; k=1, \ldots, K)$

Segundo estágio:

$$
\begin{aligned}
& y_{i k}^{*}=f\left(c_{i k}, \beta_{i}\right)+\in{ }_{i k}, \\
& y_{i k}=d_{i k} y_{i k}^{*}
\end{aligned}
$$

em que $d^{*}{ }_{i k}$ é uma variável latente representando a diferença em utilidade entre comprar ou não o i-ésimo bem; $d_{i k}$ é uma variável binária observada para representar a escolha do k-ésimo domicílio em consumir $i$-ésimo bem $\left(d_{i k}=1\right)$ ou não $\left(d_{i k}=0\right)$; $y^{*}{ }_{i k}$ é uma variável latente representando a quantidade consumida do i-ésimo produto; $y_{i k}$ é uma variável dependente observada representando a quantidade consumida com o $i$-ésimo produto; $f\left(c_{i k}, \beta_{i}\right)$ é a função de demanda; $c_{i k}$ e $z_{i k}$ são vetores de variáveis exógenas ${ }^{5} ; \beta_{i}$ e $\alpha_{i}$ são vetores de parâmetros e $\epsilon_{i k}$ e $\vartheta_{i k}$ são os erros aleatórios.

O vetor $z_{i k}$ representa as características sociodemográficas do k-ésimo domicílio que podem influenciar a propensão ao consumo do i-ésimo bem. No primeiro estágio, obtêm-se as estimativas de $\alpha_{i}$, por meio do modelo Probit. A partir das estimativas do primeiro estágio, Shonkwiller e Yen (1999) assumem que os termos de erro $\left[\epsilon_{i k} \vartheta_{i k}\right]$ sejam distribuídos como uma normal bivariada com Cov $\left(\in_{i k} \vartheta_{i k}\right)=\delta_{i}$, para cada $i$. Dessa forma, são calculadas a função de densidade de probabilidade $\phi\left(z_{i k}^{\prime} \alpha_{i}\right)$ e a função de distribuição acumulada $\Phi\left(z_{i k}^{\prime} \alpha_{i}\right)$. Por fim, $y_{i k}$ é estimado por um SUR (seemingly unrelated regression), definido da seguinte forma:

$$
y_{i k}=\Phi\left(z_{i k}^{\prime} \hat{\alpha}_{i}\right) f\left(c_{i k}, \beta_{i}\right)+\delta_{i} \phi\left(z_{i k}^{\prime} \hat{\alpha}_{i}\right)+\xi_{i k}
$$

O Procedimento de Shonkwiller e Yen (1999) é utilizado neste estudo para lidar com um problema comum na estimação de sistemas de demanda conhecido como Problema do Consumo Zero (PCZ), ou seja, grande número de domicílios que apresentam gastos nulos com algum bem particular dado o alto nível de desagregação dos produtos. O PCZ impõe algumas restrições sobre quais procedimentos econométricos podem ser adequadamente utilizados para uma precisa estimação das equações de demanda. Desse

5. As variáveis do vetor $z_{i k}$ estão listadas no Quadro 2. Além disso, o vetor $c_{i k}$ engloba as variáveis do vetor $D_{i k}$ (descrito a seguir) mais os preços e o dispêndio total $x_{r}$, e também são apresentadas no Quadro 2. modo, o Procedimento de Shonkwiller e Yen (1999), amplamente utilizado em estimações de sistemas de demanda, apresenta-se como um método eficaz para corrigir este problema.

A Teoria microeconômica não define qual a melhor forma funcional $f\left(c_{i k}, \beta_{i}\right)$ para as equações de demanda a serem estimadas. Assim, muitos autores buscaram desenvolver formas funcionais que se adequassem às propriedades postuladas na Teoria da Demanda. Neste estudo, a forma funcional é representada pelo modelo QUAIDS, desenvolvida por Banks et al. (1997). Por ser flexível, esta forma funcional possibilita impor e testar as propriedades da função de demanda (homogeneidade e simetria), sendo funções apenas dos parâmetros desconhecidos estimados no modelo. Além disso, permite incorporar os efeitos não lineares do dispêndio em sua especificação, possibilitando a obtenção de Curvas de Engel não lineares, comuns em estudos de demanda (BANKS et al., 1997).

Desse modo, o sistema de demanda a ser estimado será o seguinte:

$$
w_{i k}=\Phi\left(z_{i k}^{\prime} \hat{\alpha}_{i}\right)\left[\begin{array}{l}
\sum_{k} \theta_{i k} D_{i k}+\alpha_{i}+ \\
\sum_{j} \gamma_{i j} \ln p_{j}+\beta_{i} \ln \left(\frac{x_{r}}{a(p)}\right)+ \\
\frac{\lambda i}{b(p)}\left\{\ln \left(\frac{x_{r}}{a(p)}\right)\right\}^{2}+u_{i} \hat{v}_{k}
\end{array}\right]+
$$

$\delta_{i} \phi\left(z_{i k}^{\prime} \hat{\alpha}_{i}\right)+\xi_{i k}$

em que $w_{i k}$ é a parcela de gastos do k-ésimo domicílio com o $i$-ésimo bem; $p_{j}$ são os preços dos $n$ bens considerados; $x_{r}$ é o dispêndio total com os $n$ bens considerados no sistema; $\Phi\left(z_{i k}^{\prime} \hat{\alpha}_{i}\right)$ e $\phi\left(z_{i k}^{\prime} \hat{\alpha}_{i}\right)$ são, respectivamente, a função de distribuição acumulada e a função de densidade de probabilidade, ambas calculadas no primeiro estágio do procedimento de Shonkwiller e Yen; $D_{i k}$ é um vetor de variáveis inseridas por meio da translação demográfica que caracterizam o k-ésimo domicílio; $a(p)$ é um índice de preços translog do modelo: $\ln a(p)=a_{0}+$ $\sum_{i} \alpha_{i} \ln \left(p_{i}\right)+1 / 2 \sum_{i} \sum_{j} \gamma_{i j} \ln \left(p_{i}\right) \ln \left(p_{j}\right) ; b(p)=\prod_{k} p_{k}^{\beta k}$ é um agregador de preços Cobb-Douglas; e $\theta_{i k}, \alpha_{i}, \gamma_{i j}, \beta_{i}, \lambda_{i}, u_{i}$ e $\delta_{i}$ são os parâmetros a serem estimados.

Algumas considerações devem ser feitas sobre as variáveis $\hat{v}_{k}$ e $p_{j}$. A primeira é utilizada para corrigir o problema de endogeneidade do dispêndio $\left(x_{r}\right)$, uma vez que é possível que exista um viés de simultaneidade, devido à determinação conjunta da quantidade demandada $\left(q_{i}\right)$ e seu dispêndio $\left(x_{r}\right)$. Para solucionar 
este problema, utiliza-se a abordagem de estimação por regressão aumentada de Blundell e Robin (1999), assim como em Tafere et al. (2010), que é composta em duas etapas: na primeira, o dispêndio total é regredido num conjunto de variáveis exógenas (um vetor de características domiciliares ${ }^{6}$ e um índice de preços como instrumento adicional). Em seguida, os resíduos previstos dessa regressão $\left(\hat{v}_{k}\right)$ são incluídos nas equações do modelo QUAIDS como uma variável explicativa, junto ao dispêndio total $\left(x_{r}\right)$, permitindo-se corrigir e testar a endogeneidade do dispêndio 7 (BLUNDELL e ROBIN, 1999).

Os preços $\left(p_{j}\right)$ dos bens não são explicitamente disponibilizados na POF e, assim, são obtidos pelo cálculo dos valores unitários. Entretanto, a utilização direta de valores unitários na estimação de equações de demanda é controversa, uma vez que, como alertam Cox e Wohlgenant (1986) e Deaton (1997), eles podem ser afetados por atributos como a qualidade do bem, tornando essa variável endógena na estimação da demanda. Para solucionar este problema, utiliza-se o método proposto por Cox e Wohlgenant (1986), que consiste em estimar os preços corrigidos pelos "efeitos qualidade" regredindo a diferença entre os valores unitários e seus valores médios estaduais pelas características domiciliares ${ }^{8}$, utilizadas como proxy das preferências dos domicílios por qualidade. Assume-se que os desvios em relação aos valores unitários médios refletem "efeitos qualidade" induzidos por características domiciliares, como também fatores não sistemáticos ligados à oferta.

As elasticidades-dispêndio $\left(e_{i}\right)$, elasticidade-preço da demanda $\left(e_{i i}^{u}\right)$ e elasticidades-preços cruzadas $\left(e_{i j}^{u}\right)$ são obtidas pela diferenciação da equação (4) em relação ao logaritmo do dispêndio e dos preços, respectivamente, e podem ser escritas como:

6. Na regressão aumentada de Blundell e Robin (1999) foram utilizadas as seguintes variáveis exógenas: Metropolitana, Rural, Norte, Nordeste, Sul, Centro Oeste, Escolaridade, Sexo e o logaritmo da renda. Para uma descrição dessas variáveis, ver Quadro 2.

7. Para testar a endogeneidade do dispêndio, examina-se a significância conjunta dos parâmetros da variável $\left(\hat{v}_{k}\right)$ na equação (4).

8. As características domiciliares utilizadas no método de Cox e Wohlgenant também são apresentadas no Quadro 2 , exceto aquelas que denotam os hábitos de vida no domicílio. Além disso, optou-se por utilizar o logaritmo da renda ao invés de utilizá-la em nível.

$$
\begin{gathered}
e_{i}=\frac{\mu_{i}}{w_{i}}+1 \\
e_{i j}^{u}=\frac{\mu_{i j}}{w_{i}}-\delta_{i j}
\end{gathered}
$$

em que $\mu_{i}$ e $\mu_{i j}$ são as derivadas da parcela de gastos em relação ao logaritmo do dispêndio total e ao logaritmo dos preços, respectivamente, e $\delta_{i j}$, é denominado Delta Kronecker, que pode assumir os seguintes valores: $\delta_{i j}=0 \forall i \neq j$ e $\delta_{i j}=1 \forall i=j$.

A estimação dos parâmetros do modelo QUAIDS realizada neste estudo seguiu uma rotina de programação para o STATA, como descrita em (POI, 2008), possibilitando impor as restrições de Homogeneidade e Simetria. Segundo Yen, Lin e Smallwood (2003), a imposição da restrição de Aditividade das parcelas de gastos é garantida pela estimação de um sistema de demanda para $n-1$ bens, utilizando-se uma das categorias como "residual". Neste estudo, utilizou-se a categoria Outros orgânicos, uma vez que essa categoria representa um mix de produtos heterogêneos e apresenta baixa frequência de aquisição ${ }^{9}$. A imposição da restrição de Aditividade possibilita a recuperação dos parâmetros para esse bem, além da obtenção das elasticidades. Estima-se as equações do sistema de demanda por meio de um sistema de regressões aparentemente não relacionadas (SUR), a partir do comando NLSUR. O procedimento adotado é o IFGNLS (iterated feasible generalized non-linear least squares), equivalente às estimações por Máxima Verossimilhança. Para fazer inferência estatística sobre os valores das elasticidades aplica-se o "método delta ${ }^{10}$ ", que possibilita transformar a matriz de variância-covariância dos parâmetros estimados na matriz de variância-covariância dos parâmetros de interesse (elasticidades), permitindo-se a realização de testes de hipótese.

9. Yen e Huang (2002) especificam que, de modo geral, os pesquisadores optam por definir a categoria "outros alimentos" (muito comum em estudos de demanda) como o bem residual.

10. Para mais detalhes sobre o método delta ver Deaton (1997, p. 128-129). 


\section{Base de dados e variáveis selecionadas}

As informações utilizadas neste estudo foram retiradas dos microdados da Pesquisa de Orçamentos Familiares (IBGE, 2010a). A pesquisa, realizada pelo Instituto Brasileiro de Geografia e Estatística (IBGE), teve início no dia 19 de maio de 2008 e término no dia 18 de maio de 2009, e foi efetuada para uma amostra de 55.970 domicílios, localizados nas áreas urbanas e rurais de todo o território brasileiro. Essa pesquisa visa fornecer informações sobre as estruturas de consumo, dos gastos, dos rendimentos e parte da variação patrimonial das famílias, além de disponibilizar informações antropométricas, bem como o consumo e despesa individual. Desse modo, a Pesquisa de Orçamentos Familiares (POF) possibilita, dentre outras análises, estudar a dimensão do mercado consumidor para diversos grupos de produtos e serviços (IBGE, 2010b).
Os produtos considerados no sistema de demanda neste estudo foram escolhidos com base na classificação dos grupos alimentares da POF (IBGE, 2010c). Dentre os produtos analisados foram considerados, além de todos os alimentos orgânicos disponíveis, os seus correspondentes convencionais com características semelhantes. Nas categorias em que há maior homogeneidade entre os alimentos convencionais (Frutas, Hortaliças e Cereais/Leguminosas), todos os produtos foram considerados. Entretanto, nas categorias em que se verifica relativa diferenciação entre os alimentos convencionais (Lácteos, Carnes, Bebidas e Outros) foram selecionados apenas os alimentos convencionais que apresentaram a mesma nomenclatura que os alimentos orgânicos. Os produtos analisados são apresentados no Quadro 1.

O elevado nível de desagregação dos microdados da POF, com várias subdivisões por produto, impossibilita a estimação de sistemas de demanda para produtos

Quadro 1. Alimentos orgânicos e convencionais utilizados na estimação

\begin{tabular}{|c|c|c|c|}
\hline \multicolumn{2}{|c|}{ Frutas } & \multicolumn{2}{|c|}{ Hortaliças } \\
\hline Frutas de clima temperado & $\begin{array}{l}\text { Maçã } \\
\text { Morango } \\
\text { Uva }\end{array}$ & \multirow[t]{3}{*}{ Folhosas } & \multirow{3}{*}{\begin{tabular}{|l|} 
Agrião \\
Alface \\
Salsa \\
Couve \\
Brócolis \\
Repolho \\
Espinafre \\
Almeirão \\
Rúcula
\end{tabular}} \\
\hline Frutas de clima tropical & $\begin{array}{l}\text { Banana } \\
\text { Goiaba } \\
\text { Limão } \\
\text { Caju } \\
\text { Acerola } \\
\end{array}$ & & \\
\hline \multicolumn{2}{|c|}{ Cereais e leguminosas } & & \\
\hline Cereal & Arroz & \multirow[t]{4}{*}{ Frutosas } & \multirow{4}{*}{\begin{tabular}{|l} 
Jiló \\
Pepino \\
Pimentão \\
Tomate \\
Vagem \\
\end{tabular}} \\
\hline Leguminosa & Feijão & & \\
\hline \multicolumn{2}{|c|}{ Lácteos } & & \\
\hline \multirow{2}{*}{\multicolumn{2}{|c|}{$\begin{array}{l}\text { Creme de leite } \\
\text { Leite de vaca } \\
\text { Queijo minas frescal } \\
\text { Iogurte }\end{array}$}} & & \\
\hline & & \multirow[t]{2}{*}{ Tuberosas e outras } & \multirow[t]{2}{*}{$\begin{array}{l}\text { Batata-inglesa } \\
\text { Beterraba } \\
\text { Cenoura }\end{array}$} \\
\hline \multicolumn{2}{|c|}{ Bebidas } & & \\
\hline \multirow[t]{2}{*}{ Alcóolicas } & \multirow{2}{*}{$\begin{array}{l}\text { Aguardente de cana } \\
\text { Aguardente de arroz } \\
\text { Vinho de uva e outros }\end{array}$} & \multicolumn{2}{|c|}{ Carnes } \\
\hline & & \multirow[t]{2}{*}{ Bovina } & \multirow{2}{*}{$\begin{array}{l}\text { Alcatra } \\
\text { Carne bovina de primeira } \\
\text { Chã de dentro } \\
\text { Contra-filé } \\
\text { Lagarto } \\
\text { Patinho }\end{array}$} \\
\hline Não alcóolicas & $\begin{array}{l}\text { Sucos engarrafados } \\
\text { Suco encartonados } \\
\text { Sucos para viagem } \\
\text { Sucos em saco plástico }\end{array}$ & & \\
\hline \multicolumn{2}{|c|}{ Outros } & \multirow[t]{2}{*}{ Frango } & \multirow[b]{2}{*}{$\begin{array}{l}\text { Frango inteiro } \\
\text { Frango congelado } \\
\text { Cortes de frango } \\
\text { Coxa e sobrecoxa } \\
\text { Peito de frango }\end{array}$} \\
\hline $\begin{array}{l}\text { Café } \\
\text { Ovo de galinha } \\
\text { Mel de abelha } \\
\text { Erva mate } \\
\text { Proteína de soja } \\
\text { Açúcar }\end{array}$ & & & \\
\hline
\end{tabular}

Fonte: Elaborado pelos autores a partir das informações da POF (2008-2009) (IBGE, 2010a). 
Tabela 1. Proporção de domicílios com consumo zero de alimentos convencionais e orgânicos, 2009

\begin{tabular}{lcc}
\hline \multirow{2}{*}{ Categorias } & \multicolumn{2}{c}{ Domicílios com consumo zero (\%) } \\
\cline { 2 - 3 } & Alimentos Convencionais & Alimentos Orgânicos \\
\hline Frutas & 61,52 & 99,88 \\
Hortaliças & 51,39 & 99,68 \\
Cereais/Leguminosas & 51,73 & 99,80 \\
Lácteos & 44,96 & 99,40 \\
Bebidas & 92,93 & 99,75 \\
Carnes & 51,41 & 99,74 \\
Outros & 40,04 & 99,77 \\
\hline
\end{tabular}

Fonte: Elaborado pelos autores a partir dos dados da POF 2008/2009 (IBGE, 2010a).

isolados. Complementando este quadro, a baixíssima frequência da aquisição de alimentos orgânicos nos domicílios brasileiros (Tabela 1) impossibilitou uma análise mais individualizada destes bens. Assim, foi necessário reunir os vários subgrupos de produtos em categorias mais amplas, que representassem conjuntamente as principais características dos produtos incluídos. Por exemplo, o agregado Frutas é composto pela junção dos subgrupos "frutas de clima temperado" e "frutas de clima tropical". Embora o procedimento de agregação de bens seja necessário, buscou-se criar categorias de produtos mais desagregadas possível, de modo a possibilitar que as elasticidades estimadas descrevessem de forma mais acurada o comportamento dos consumidores brasileiros frente a variações nos preços relativos e no dispêndio. Por exemplo, procurou-se separar frutas de hortaliças, pois espera-se que o padrão de substituição seja diferente nestes grupos.

A baixa frequência de aquisição está associada ao modo como os dados das POF's são obtidos. Isso porque esses dados são obtidos por meio de questionários que investigam o comportamento de consumo dos domicílios em um período específico. No caso dos alimentos e bebidas, as quantidades desses bens foram pesquisadas segundo um período de sete dias (IBGE, 2010c). Desse modo, é bastante provável a inclusão de consumidores que consomem determinado bem, mas que não o adquiriram na semana da pesquisa devido ao fato de a aquisição do bem ter ocorrido em período distinto ao da semana da pesquisa ou devido à preferência por estoques. $\mathrm{O}$ outro fator que explica o consumo zero decorre da impossibilidade de que os consumidores adquiram todos os produtos considerados na pesquisa, uma vez que o universo de produtos pesquisados numa POF é imenso. Assim, soluções de canto para o problema de maximização da utilidade são naturalmente observados para todos os consumidores e, desse modo, o consumo zero é uma escolha das famílias dadas suas preferências e restrição orçamentária.

As categorias agregadas foram obtidas considerando-se os diferentes pesos que os diversos tipos de alimentos têm no orçamento do consumidor. Desse modo, os pesos foram calculados dividindo-se a despesa total do produto correspondente pela despesa total com todos os produtos incluídos na categoria definida. $\mathrm{O}$ valor gasto e a quantidade adquirida de forma agregada foram encontrados por meio do somatório dos valores gastos e quantidades com cada bem que compôs a categoria de interesse, respectivamente. O preço agregado é dado pelo somatório do produto dos pesos calculados pelos preços de cada bem. Após estes procedimentos, as categorias agregadas consideradas neste estudo foram Frutas, Hortaliças, Cereais/ Leguminosas, Lácteos, Carnes, Bebidas e Outros, sendo que cada uma delas é agrupada entre alimentos orgânicos e convencionais.

Os vetores de variáveis sociodemográficas que permitem captar alterações nos padrões de consumo entre os domicílios no que se refere à localização e composição domiciliar, além dos hábitos de vida são apresentados no Quadro 2. 
Quadro 2. Variáveis presentes nos vetores $z_{i k}$ e $D_{i k}$

\begin{tabular}{|c|c|}
\hline Variáveis & Descrição \\
\hline \multicolumn{2}{|l|}{ Localização domiciliar } \\
\hline Rural & Domicílio localizado em zona rural $=1$; caso contrário $=0$ \\
\hline Metropolitana & Domicílio localizado em área metropolitana $=1$; caso contrário $=0$ \\
\hline Norte & Domicílio localizado na região Norte $=1$; caso contrário $=0$ \\
\hline Nordeste & Domicílio localizado na região Nordeste $=1$; caso contrário $=0$ \\
\hline Sul & Domicílio localizado na região $S u l=1$; caso contrário $=0$ \\
\hline Centro-Oeste & Domicílio localizado na região Centro Oeste $=1$; caso contrário $=0$ \\
\hline \multicolumn{2}{|l|}{ Composição Domiciliar } \\
\hline Renda* & Renda domiciliar mensal \\
\hline Sexo & Pessoa de referência do sexo feminino $=1$; caso contrário $=0$ \\
\hline Escolaridade & Anos de estudo da pessoa de referência da família \\
\hline Idade & Idade da pessoa de referência da família \\
\hline Idade ao quadrado & Idade da pessoa de referência da família elevada ao quadrado \\
\hline \multicolumn{2}{|l|}{ Hábitos de Vida } \\
\hline Atividade Física & Gastos com atividades físicas no domicílio $=1 ;$ caso contrário $=0$ \\
\hline Avaliação alimentar & Sempre consome o tipo de alimento que quer $=1$; caso contrário $=0$ \\
\hline
\end{tabular}

Nota: * Variável utilizada no primeiro estágio e na correção da endogeneidade dos preços e do dispêndio.

Fonte: Delineamento do estudo.

Dentre os 55.970 domicílios entrevistados pela POF, 224 não informaram alguma das variáveis demográficas consideradas. Também foram retiradas 1.134 observações, cujos preços finais, estimados através do procedimento de Cox e Wohlgenant (1986), apresentaram valores muito discrepantes. Além disso, 10.660 domicílios não apresentaram o consumo de nenhum dos bens analisados e também foram retirados da amostra. A amostra final possui 43.952 observações, correspondente aos domicílios que declararam o consumo de pelo menos um dos bens (incluindo o bem residual).

\section{Resultados e discussão}

\subsection{Primeiro estágio ${ }^{11}$}

A partir do primeiro estágio do procedimento de Shonkwiller e Yen (1999), são obtidas as estimativas que definem a propensão à aquisição dos bens analisados mediante a influência de fatores sociodemográficos que são definidos no vetor de variáveis explicativas no modelo Probit. Dentre as variáveis que caracteri-

11. Nesta seção, são apresentados resumidamente os efeitos marginais estatisticamente significativos estimados para as categorias de alimentos orgânicos. zam a "Composição Domiciliar", pôde-se verificar que, nos domicílios em que há uma mulher como pessoa de referência, a probabilidade de aquisição de alimentos orgânicos foi significativa apenas para a categoria Outros. Este resultado mostra que esses domićlios têm probabilidade aproximada de 0,25 ponto percentual (p.p.) maior de consumir alimentos incluídos na categoria Outros Orgânicos do que domicílios cuja pessoa de referência é um homem.

A escolaridade foi a variável de controle que apresentou a maior quantidade de efeitos marginais significativos para as categorias de alimentos orgânicos, evidenciando que os domicílios em que a pessoa de referência tem maior estoque educacional têm maior probabilidade de consumo de orgânicos. Para cada ano adicional na escolaridade da pessoa de referência, mantidas constantes as demais variáveis, a propensão à aquisição é aumentada em 0,29 ponto percentual (p.p.) para Frutas Orgânicas, 1,66 p.p. para Hortaliças Orgânicas, 0,25 p.p. para Cereais/Leguminosas Orgânicas, 0,63 p.p. para Bebidas Orgânicas e 0,18 p.p. para a categoria Outros Orgânicos. Além disso, a escolaridade impacta na decisão de consumo de alimentos orgânicos em magnitude superior à própria renda per capita, que apresentou efeitos marginais positivos para as categorias Frutas, Hortaliças, Lácteos e Bebidas Orgânicas. 
Quanto aos efeitos marginais da variável idade, constatou-se que, a cada ano de aumento na idade da pessoa de referência, a probabilidade de consumo de Hortaliças, Cereais/Leguminosas e Outros Orgânicos aumenta em 0,02 p.p., 0,006 p.p. e 0,006 p.p., respectivamente.

Em relação às variáveis do grupo de "Localização Domiciliar", notou-se um efeito positivo da variável Rural sobre a probabilidade de aquisição de três categorias de orgânicos: Hortaliças (0,52 p.p.), Lácteos (0,49 p.p.) e Cereais/Leguminosas (0,16 p.p.). Em relação aos efeitos marginais regionais, a influência sobre a propensão ao consumo de orgânicos é bastante limitada. A região Sul destaca-se por apresentar maior probabilidade de aquisição de Hortaliças Orgânicas do que a região base (Sudeste), com 0,64 ponto percentual. Em contraste, a região Norte destaca-se por apresentar menor probabilidade de aquisição em relação à região base para as categorias Frutas $(-0,09$ p.p.), Hortaliças (-0,34 p.p.) e Lácteos Orgânicos (-0,65 p.p.).

Para as variáveis que denotam os "Hábitos de Vida" no domicílio, verificou-se que a Avaliação Alimentar não é estatisticamente diferente de zero nas categorias de alimentos orgânicos, exceto a categoria Hortaliças (0,32 p.p.). No caso de Atividade Física, observa-se que, nos domicílios que efetuaram dispêndios com atividades físicas, a probabilidade de consumo de Hortaliças, Carnes e Outros Orgânicos se eleva em torno de 0,63 p.p., 0,65 p.p., e 0,32 p.p. respectivamente.

A partir das estimativas do primeiro estágio, são calculadas a função de densidade de probabilidade $\phi\left(z_{i k}^{\prime} \alpha_{i}\right)$ e a função de distribuição acumulada $\Phi\left(z_{i k}^{\prime} \alpha_{i}\right)$ utilizadas no segundo estágio de estimação, cujos resultados são apresentados a seguir.

\subsection{Segundo estágio}

A partir do segundo estágio do sistema de demanda foram estimados 312 parâmetros. Dentre eles, 130 são relacionados à cesta de bens, 156 são relacionados às variáveis demográficas, 13 são obtidos devido à aplicação do procedimento de Shonkwiller e Yen e 13 são obtidos pela incorporação da variável de correção de endogeneidade do dispêndio. Desses, 67\% foram significativos considerando-se o nível de significância igual de $10 \%$.

Antes de apresentar os resultados referentes às elasticidades, verificam-se as estimativas dos parâmetros $\lambda$, referentes à forma quadrática do dispêndio no sistema de equações e $u$, usado para corrigir a endogeneidade do dispêndio. A Tabela 2 apresenta os resultados para os testes de significância conjunta desses parâmetros. A análise da estatística $\chi^{2}$ permite inferir que as hipóteses nulas de linearidade e exogeneidade do dispêndio total são rejeitadas, para o sistema como um todo, ao nível de significância de $1 \%$. Desse modo, os resultados indicam que o sistema de demanda será apropriadamente estimado considerando-se a especificação QUAIDS com correção da endogeneidade do dispêndio.

Após a estimação dos coeficientes do sistema de demanda, eles são utilizados para calcular o grau de sensibilidade dos consumidores mediante variações nos preços e no dispêndio total com a cesta de bens considerada, dadas pelas elasticidades-dispêndio e elasticidades-preço próprias, respectivamente (ver equações 5 e 6 ). As elasticidades-dispêndio $\left(e_{i}\right)$ e elasticidades-preço próprias $\left(e_{i i}^{u}\right)$ marshallianas são apresentadas na Tabela 3, considerando-se os pontos médios da amostra, para as 14 categorias analisadas. Verifica-se que as elasticidades estimadas são estatisticamente significativas ao nível de $1 \%$ de probabilidade para todas as categorias analisadas, exceto a elasticidade-preço para Outros Orgânicos. Além disso, nota-se que todas as categorias apresentam elasticidades-preço próprias negativas, em conformidade com a teoria microeconômica, e elasticidades-dispêndio positivas - a exceção, novamente é a categoria Outros Orgânicos, estimada como o bem residual.

Com relação às elasticidades-dispêndio, pode-se verificar que todas as categorias pertencentes ao grupo de alimentos orgânicos apresentam elasticidade supe-

Tabela 2. Teste de Wald para a significância conjunta dos parâmetros $\lambda$ e $u$ no sistema de demanda, 2009

\begin{tabular}{ccc}
\hline Hipótese nula & $\chi^{2}$ & Prob $>\chi^{2}$ \\
\hline$\lambda_{1}=\lambda_{2}=\ldots=\lambda_{n}=0$ & 220,23 & 0,000 \\
$u_{1}=u_{2}=\ldots=u_{n}=0$ & 80,54 & 0,000 \\
\hline
\end{tabular}

Fonte: Resultados da pesquisa. 
Tabela 3. Elasticidades-dispêndio $\left(e_{i}\right)$ e elasticidades-preço próprias $\left(e_{i i}^{u}\right)$ marshallianas, 2009

\begin{tabular}{lcccc} 
& \multicolumn{3}{c}{ Tipo de alimento } \\
\cline { 2 - 5 } & \multicolumn{3}{c}{ Orgânicos } & \multicolumn{2}{c}{ Convencionais } \\
\cline { 2 - 5 } & $\mathbf{e}_{\mathbf{i}}$ & $e_{i i}^{u}$ & $\mathbf{e}_{\mathbf{i}}$ & $e_{i i}^{u}$ \\
\hline Frutas & $1,310^{* * *}$ & $-2,312^{* * *}$ & $0,965^{* * *}$ & $-1,106^{* * *}$ \\
Hortaliças & $1,241^{* * *}$ & $-3,646^{* * *}$ & $0,940^{* * *}$ & $-1,098^{* * *}$ \\
Cereais e Leguminosas & $1,327^{* * *}$ & $-1,459^{* * *}$ & $1,051^{* * *}$ & $-1,056^{* * *}$ \\
Lácteos & $1,325^{* * *}$ & $-2,338^{* * *}$ & $1,027^{* * *}$ & $-1,041^{* * *}$ \\
Bebidas & $1,045^{* * *}$ & $-1,412^{* * *}$ & $0,906^{* * *}$ & $-1,308^{* * *}$ \\
Carnes & $1,687^{* * *}$ & $-1,309^{* * *}$ & $1,075^{* * *}$ & $-1,055^{* * *}$ \\
Outros & $-3,127^{* * *}$ & $-1,223$ & $0,853^{* * *}$ & $-1,038^{* * *}$ \\
\hline
\end{tabular}

Nota: nível de significância ***1\%.

Fonte: Resultados da pesquisa.

rior à unidade em módulo, evidenciando que são bens superiores ou de luxo. Além disso, pode-se notar que os alimentos orgânicos são mais sensíveis a variações no dispêndio do que alimentos convencionais considerados na amostra. Isso significa que um aumento de 1 ponto percentual no dispêndio com os alimentos considerados promove um aumento no consumo de alimentos orgânicos em magnitude superior ao dos alimentos convencionais. Desse modo, espera-se que elevações na renda e, consequentemente, no dispêndio da população brasileira, aumentem o consumo de orgânicos no País vis-à-vis suas contrapartes convencionais. Ressalta-se que a renda mostra-se de grande importância para explicar a quantidade adquirida dos bens, principalmente os orgânicos, que são mais caros. Para o grupo de alimentos convencionais, nota-se que as elasticidades-dispêndio apresentam magnitudes muito próximas da unidade, havendo predominância de bens normais entre as categorias estudadas. Dentre as categorias de alimentos convencionais que apresentam elasticidade-dispêndio maior que 1, a categoria Carnes destaca-se com a maior magnitude.

Quanto aos preços, verifica-se que a demanda é elástica em todas as categorias de alimentos em ambos os grupos analisados. Ressalta-se que, conforme esperado, os alimentos orgânicos apresentam sensibilidade superior a variações nos preços em todas as categorias quando comparados aos convencionais. Em especial, destacam-se as categorias Frutas, Hortaliças e Lácteos, em que as elasticidades-preços (em módulo) na categoria de orgânicos são mais do que o dobro das suas contrapartes convencionais. Estes resultados são semelhantes aos encontrados em estudos anteriores (LIN et al.,
2009; KASTERIDIS e YEN, 2012) que compararam elasticidades-preço entre alimentos orgânicos e convencionais. Conforme destacado por Schröck (2010), há uma tendência consolidada entre estudos que investigam a relação entre orgânicos e convencionais em indicar que a demanda por alimentos orgânicos é mais elástica do que a demanda por alimentos convencionais. Este resultado decorre do fato de os orgânicos apresentarem preços elevados em relação aos alimentos convencionais e possuírem pequena parcela de mercado (LIN et al., 2009). Assim, aumentos nos preços de alimentos orgânicos tendem a reduzir a quantidade consumida em uma proporção superior aos convencionais. Por outro lado, pode-se inferir que uma redução nos preços dos orgânicos influencia positivamente o consumo desses bens em magnitude superior aos convencionais.

De modo geral, verifica-se que os bens em análise são mais sensíveis a variações nos preços do que no dispêndio, principalmente entre os alimentos orgânicos. A exceção é a categoria Carnes Convencionais.

\subsubsection{Elasticidades-preços cruzadas}

Para facilitar a visualização, as elasticidades-preço cruzadas são divididas em dois subgrupos. A Tabela 4 apresenta as elasticidades-preço cruzadas dentro dos grupos de alimentos orgânicos e convencionais, respectivamente. Já a Tabela 5 apresenta as elasticidades-preço cruzadas entre alimentos orgânicos e convencionais.

Na parte superior da Tabela 4, são apresentadas as relações de complementariedade e substitutibilidade bruta entre os alimentos orgânicos. São observadas 22 
Tabela 4. Elasticidades-preço cruzadas $\left(e_{i i}^{u}\right)$ marshallianas entre alimentos orgânicos e entre alimentos convencionais, 2009

\begin{tabular}{|c|c|c|c|c|c|c|c|}
\hline \multirow{2}{*}{$\begin{array}{c}\text { Categorias } \\
\text { Orgânicos }\end{array}$} & \multicolumn{7}{|c|}{ Orgânicos } \\
\hline & Frutas & Hortaliças & Cereais/Leguminosas & Lácteos & Bebidas & Carnes & Outros \\
\hline Frutas & - & $3,291^{* * *}$ & $0,936^{* *}$ & $-2,589^{*}$ & $-0,474$ & $1,075^{*}$ & 0,653 \\
\hline Hortaliças & $0,374^{* * *}$ & - & $0,190^{* *}$ & 0,007 & $0,591^{* * *}$ & $0,609^{* * *}$ & $-0,771^{* * *}$ \\
\hline Cereais/Leguminosas & $0,197^{* *}$ & $0,308^{* *}$ & - & $1,102^{* * *}$ & 0,133 & 0,200 & $-0,860 * * *$ \\
\hline Lácteos & $-0,050^{* *}$ & 0,001 & $0,124^{* * *}$ & - & $-0,044$ & 0,077 & $0,488^{* * *}$ \\
\hline Bebidas & $-0,059$ & $0,641^{* * *}$ & 0,081 & $-0,288$ & - & $-0,232$ & $0,388^{* *}$ \\
\hline Carnes & $0,073^{* *}$ & $0,337^{* * *}$ & 0,086 & 0,161 & $-0,113$ & - & $-0,242$ \\
\hline \multirow[t]{2}{*}{ Outros } & 0,103 & $-1,112^{* * *}$ & $-0,883$ & $4,734^{* * *}$ & 0,499 & $-0,658$ & - \\
\hline & \multicolumn{7}{|c|}{ Convencionais } \\
\hline Convencionais & Frutas & Hortaliças & Cereais/Leguminosas & Lácteos & Bebidas & Carnes & Outros \\
\hline Frutas & - & $0,043^{* * *}$ & 0,003 & $0,023^{* * *}$ & $0,019^{* * *}$ & $0,058^{* * *}$ & $-0,000$ \\
\hline Hortaliças & $0,027^{* * *}$ & - & $0,023^{* * *}$ & $0,080^{* * *}$ & 0,001 & 0,000 & $0,024^{* * *}$ \\
\hline Cereais/Leguminosas & $-0,004$ & 0,002 & - & $-0,019^{* * *}$ & $-0,004^{* * *}$ & 0,003 & $0,025^{* * *}$ \\
\hline Lácteos & 0,002 & $0,029 * * *$ & $-0,011^{* * *}$ & - & $-0,000$ & $-0,008^{* * *}$ & 0,002 \\
\hline Bebidas & $0,562^{* * *}$ & 0,129 & $-0,198^{* * *}$ & 0,067 & - & $0,398^{*}$ & 0,003 \\
\hline Carnes & $0,007^{* * *}$ & $-0,013^{* * *}$ & $-0,000$ & $-0,019^{* * *}$ & $0,002^{*}$ & - & 0,002 \\
\hline Outros & $0,004^{*}$ & $0,018^{* * *}$ & $0,054^{* * *}$ & $0,035^{* * *}$ & $-0,001$ & 0,050 & - \\
\hline
\end{tabular}

Nível de significância: ${ }^{* * *} 1 \%, * * 5 \%, * 10 \%$.

Fonte: Resultados da pesquisa.

relações significativas, sendo que cinco são de complementaridade entre as categorias de orgânicos, e 17 de substituição. Verifica-se que as relações de complementaridade e substituição são iguais entre quase todas as categorias, exceto entre Outros e as demais; ou seja, o padrão predominante de substituição pode ser observado entre os pares de categorias. Embora se possa verificar uma forma de "simetria" quanto às relações, adverte-se que as magnitudes são diferentes: por exemplo, uma variação positiva de um ponto percentual nos preços das Hortaliças Orgânicas tende a aumentar a quantidade demandada de Frutas Orgânicas em cerca 3,3 p.p., entretanto, o mesmo impacto sobre as Hortaliças Orgânicas não é verificado quando os preços das Frutas Orgânicas se elevam em 1 p.p.; nesse caso, a quantidade demandada aumenta em 0,37 ponto percentual. De modo geral, esses resultados indicam que os alimentos orgânicos tendem a não serem consumidos conjuntamente, notando-se uma relação de rivalidade na aquisição desses bens. Os preços elevados são um dos fatores que podem ajudar a explicar este comportamento, levando o consumidor brasileiro de orgânicos a escolher apenas um produto/categoria para adquirir.

Semelhantes aos orgânicos, os alimentos convencionais apresentam maior proporção de relações de substituição entre si. Desse modo, compreende-se que tanto variações nos preços dos alimentos orgânicos quanto de suas contrapartes convencionais tendem a fazer com que os consumidores optem por substituir os bens dentro do mesmo grupo, dadas suas respectivas classificações.

A Tabela 5 apresenta os resultados referentes às elasticidades-preço cruzadas entre alimentos orgânicos e convencionais. Conforme esperado, não se verifica uma relação de substituição/complementaridade simétrica entre os dois tipos de alimentos. As elasticidades-preço cruzadas entre os pares de categorias para os bens semelhantes dos dois grupos de alimentos são apresentadas nas diagonais principais e em negrito. Conforme pode ser observado, variações nos preços das categorias convencionais tendem a impactar no consumo dos orgânicos em uma proporção muito maior do que a mesma variação em sentido inverso. Pode-se notar que, quando há variações nos preços das categorias convencionais, as únicas relações de substituição significativas são entre Hortaliças e Outros $(8,14$ e 134 , respectivamente). Ressalta-se que estas duas categorias de alimentos orgânicos são as mais representativas na amostra, simbolizando que, quanto maior tende a ser consumo de orgânicos, maiores tendem a ser as relações de substituição entre esses e os convencionais. 
Tabela 5. Elasticidades-preço cruzadas $\left(e_{i i}^{u}\right)$ marshallianas entre alimentos orgânicos e convencionais, 2009

\begin{tabular}{|c|c|c|c|c|c|c|c|}
\hline \multirow{2}{*}{$\begin{array}{c}\text { Categorias } \\
\text { Orgânicos }\end{array}$} & \multicolumn{7}{|c|}{ Convencionais } \\
\hline & Frutas & Hortaliças & Cereais/Leguminosas & Lácteos & Bebidas & Carnes & Outros \\
\hline Frutas & $-30,456^{* * *}$ & $-19,552$ & $-111,80^{* * *}$ & 0,8856 & $-9,4672 * * *$ & $-40,054^{*}$ & $62,568^{* * *}$ \\
\hline Hortaliças & $-0,8638$ & $8,1451^{* *}$ & $10,147^{* *}$ & $-15,793^{* * *}$ & $2,6577^{* *}$ & $-0,8679$ & $-35,115^{* * *}$ \\
\hline Cereais/Leguminosas & $-2,2643$ & $-3,2724$ & $-4,9348$ & $-27,861^{* * *}$ & $-2,6096^{* *}$ & $-13,308$ & $-28,776$ \\
\hline Lácteos & $-5,8328^{* * *}$ & $3,1381^{* * *}$ & $-6,8228^{* * *}$ & $-4,9106^{* * *}$ & $0,5551^{* *}$ & $-8,3629 * * *$ & $-2,7575^{* *}$ \\
\hline Bebidas & 1,9601 & $-6,0609^{*}$ & $-7,5824^{*}$ & 1,5571 & 0,0935 & $-4,0751$ & 6,4553 \\
\hline Carnes & $-8,7950^{* * *}$ & $-18,969^{* * *}$ & $-15,473^{* * *}$ & $-27,209^{* * *}$ & $2,0874^{* * *}$ & $-44,789 * * *$ & $-29,617^{* * *}$ \\
\hline \multirow[t]{2}{*}{ Outros } & $49,753^{* * *}$ & $56,311^{* * *}$ & $144,69^{* * *}$ & $149,99 * * *$ & $6,4423^{* * *}$ & $199,31^{* * *}$ & $134,00^{* * *}$ \\
\hline & \multicolumn{7}{|c|}{ Orgânicos } \\
\hline Convencionais & Frutas & Hortaliças & Cereais/Leguminosas & Lácteos & Bebidas & Carnes & Outros \\
\hline Frutas & $-0,0001^{*}$ & 0,0000 & 0,0000 & $-0,0011^{* * *}$ & 0,0001 & 0,0000 & 0,0001 \\
\hline Hortaliças & $-0,0000$ & $0,0003^{* * *}$ & 0,0000 & $0,0010^{* * *}$ & $-0,0001^{*}$ & $-0,0002$ & $-0,0002^{* * *}$ \\
\hline Cereais/Leguminosas & $-0,0001^{* * *}$ & $0,0002^{* * *}$ & $0,0001^{*}$ & $-0,0002$ & $-0,0000$ & $0,0002^{* * *}$ & $0,0001^{* * *}$ \\
\hline Lácteos & $0,0000^{* * *}$ & $-0,0001^{* * *}$ & $-0,0000^{* * *}$ & $0,0001^{* * *}$ & $0,0000^{*}$ & $0,0001^{* * *}$ & $-0,0001^{* * *}$ \\
\hline Bebidas & $-0,0014^{* * *}$ & $0,0037^{* *}$ & $-0,0020^{* *}$ & $0,0048^{* * *}$ & 0,0000 & $0,0052^{* * *}$ & $0,0048^{* * *}$ \\
\hline Carnes & 0,0000 & 0,0000 & 0,0000 & $0,0001^{* *}$ & $-0,0000$ & $-0,0002^{* * *}$ & $0,0001^{* * *}$ \\
\hline Outros & $0,0001^{* * *}$ & $-0,0004^{* * *}$ & $-0,0001^{*}$ & $0,0002^{* * *}$ & $0,0001^{* * *}$ & $-0,0004$ & $-0,0001^{* * *}$ \\
\hline
\end{tabular}

Nível de significância: ${ }^{* * *} 1 \%,{ }^{* *} 5 \%,{ }^{*} 10 \%$.

Fonte: Resultados da pesquisa.

Por outro lado, verifica-se que há forte relação de complementaridade para Frutas, Lácteos e Carnes $(-30,45$, $-4,91$ e $-44,79$, respectivamente). Esses resultados indicam que a aquisição de alimentos orgânicos no Brasil é bastante dependente dos convencionais, uma vez que elevações nos preços dos convencionais - principalmente Frutas e Carnes - reduzem significativamente a quantidade consumida de suas contrapartes orgânicas, para quem consome ambos os tipos de alimentos.

Ao se analisar o comportamento dos consumidores mediante alterações nos preços dos alimentos orgânicos, pode-se notar que é praticamente nula sua influência sobre a aquisição de alimentos convencionais. Contudo, destaca-se que esse resultado deixa evidente uma importante característica do mercado de orgânicos. Constata-se que há forte fidelidade dos consumidores aos produtos orgânicos. Adotando a interpretação dada por Glaser e Thompson (1998), pode-se dizer que as assimetrias encontradas entre as elasticidades-preço cruzadas indicam que é relativamente difícil induzir consumidores habituados a adquirir produtos orgânicos a "reverterem" seus hábitos de consumo, trocando produtos orgânicos por convencionais, mesmo os preços dos orgânicos sendo mais elevados. Uma breve análise da Tabela 4 auxilia a sustentar esta afirmação. Conforme visto anteriormente, variações nos preços dos alimentos orgânicos tendem a fazem com que os consumidores os substituam entre si.

Com relação às Hortaliças (principal categoria de orgânicos consumida na amostra), verifica-se que há relação de substituição quando se alteram os preços relativos em ambos os sentidos. Verifica-se grande aumento no consumo de Hortaliças Orgânicas quando se elevam os preços das Hortaliças Convencionais (uma variação positiva de um ponto percentual nos preços da categoria convencional tende a aumentar a quantidade demandada da categoria orgânica em 8,14 pontos percentuais), e nota-se que o contrário não ocorre, ou seja, aumentos nos preços das Hortaliças Orgânicas causam pouca variação sobre a quantidade adquirida de sua contraparte convencional (uma elevação de 100 pontos percentuais nos preços da categoria orgânica tende a elevar a quantidade consumida da categoria convencional em 0,03 ponto percentual). Entre os estudos que também analisaram a relação entre hortaliças convencionais e orgânicas, também foram encontrados resultados semelhantes quanto às assimetrias das magnitudes entre as elasticidades-preço cruzadas (FORMOUZI et al. 2012; GLASER e THOMPSON, 1998; KASTERIDIS e YEN, 2012).

Tanto as Frutas quanto as Carnes apresentaram comportamento semelhante ao das Hortaliças; porém, 
constata-se relação de complementaridade entre elas e suas contrapartes orgânicas, sendo as elasticidades $(-30,45)$ e $(-0,0001)$ para Frutas e $(-44,79)$ e $(-0,0002)$ para Carnes. Novamente, destaca-se o grande impacto que variações nos preços das categorias convencionais exercem sobre o consumo de suas contrapartes orgânicas, corroborando a hipótese inicial deste estudo.

Entre as categorias Lácteos e Outros, não se verifica concordância em relação ao padrão de substituição ou complementaridade entre os pares analisados, pois os sinais das elasticidades-preço são contrários, $(-4,91)$ e $(0,0001)$ para Lácteos e $(134)$ e $(-0,0001)$ para Outros. Em estudos internacionais sobre a demanda por leite orgânico e convencional, Glaser e Thompson (2000) e Dhar e Foltz (2005) também verificaram essa assimetria quanto às magnitudes das elasticidades-preço cruzadas. Quanto à categoria Cereais/Leguminosas, verifica-se que há relação unilateral de substituição entre seus pares, ou seja, um aumento no preço dos Cereais/ Leguminosas Orgânicas tende a elevar, embora em magnitude baixíssima, o consumo de sua contraparte convencional.

De modo geral, compreende-se que o mercado de alimentos orgânicos ainda é formado por poucos consumidores fiéis e pouco influencia o mercado de seu rival. Além disso, verifica-se que os alimentos orgânicos ainda não são vistos pelos consumidores como substitutos dos convencionais na maioria dos casos, com a exceção sendo a categoria de hortaliças. Esse fato decorre provavelmente da grande diferença de preços ainda existente entre orgânicos e convencionais na maioria dos $\operatorname{casos}^{12}$ e pela pouca oferta de orgânicos em grande parte das cidades do País.

\section{Considerações finais}

Este estudo apresenta contribuição inédita à literatura nacional sobre alimentos orgânicos por realizar a estimação de um sistema de demanda para alimentos orgânicos e suas contrapartes convencionais, apresentando as elasticidades-preço próprias e cruzadas, e

12. Os dados utilizados neste estudo mostram que essas diferenças são, em média, maiores do que $50 \%$ para a maioria dos produtos, podendo chegar a mais de $100 \%$ em alguns casos. Azzolini et al. (2007) também observaram que o preço pago pelo produto orgânico é, em média, 34,51\% maior do que em relação ao convencional. as elasticidades-dispêndio. As hipóteses sobre o comportamento dos domicílios quanto à demanda de alimentos orgânicos foram, de modo geral, confirmadas. Verificou-se que, uma vez que o domicílio opta por consumir um alimento orgânico, a renda (dispêndio) mostra-se de grande importância, determinando a quantidade a ser adquirida. Notou-se também, por meio das elasticidades-dispêndio, que os alimentos orgânicos podem ser classificados como bens superiores ou de luxo.

Com relação aos preços, pôde-se verificar que os domicílios se mostraram mais sensíveis a variações nos preços dos orgânicos do que em relação ao dispêndio. Além disso, constatou-se que os consumidores também são mais sensíveis a variações nos preços dos orgânicos do que dos convencionais. Desse modo, compreende-se que políticas de incentivo à ampliação do consumo de alimentos orgânicos podem ser mais eficazes se atuarem sobre a redução de seus preços.

Verificou-se também a assimetria entre as relações de substituição/complementaridade entre orgânicos e convencionais. Constatou-se, por meio da análise das elasticidades-preço cruzadas, que variações nos preços das categorias convencionais tendem a impactar o consumo dos orgânicos em uma proporção muito maior do que a mesma variação em sentido inverso. A análise dos efeitos de alterações nos preços dos alimentos orgânicos evidencia que há uma forte relação de fidelização dos consumidores aos produtos orgânicos. Esses resultados permitem concluir que é relativamente difícil induzir consumidores habituados a adquirirem produtos orgânicos a "reverterem" seus hábitos de consumo, trocando produtos orgânicos por convencionais, mesmo os preços dos orgânicos sendo mais elevados. Além disso, pôde-se notar que os alimentos orgânicos ainda não são vistos pelos consumidores como substitutos dos convencionais na maioria dos casos.

Apesar de os resultados encontrados possibilitarem melhor entendimento dos fatores econômicos que influenciam o consumo de alimentos mais saudáveis e ambientalmente sustentáveis, uma ressalva deve ser feita quanto à limitação deste estudo, que foi a necessidade de agregar os bens em categorias mais amplas, dado o problema do consumo zero. Desse modo, sugere-se, para estudos futuros, tentar estimar um sistema de demanda mais desagregado para alimentos orgânicos e suas contrapartes convencionais, obtendo-se elasticidades por produto, ampliando o campo de compreensão deste mercado promissor. 


\section{Referências}

ARCHANJO, L. R., BRITO, K. F. W. e SAUERBECK $\mathrm{S}$. Os alimentos orgânicos em Curitiba: consumo e significado. Cadernos de debate, v. 8, p. 1-6, 2001.

ASSIS, R. L., AREZZO, D. C. e DE-POLLI, H. Consumo de produtos da agricultura orgânica no estado do Rio de Janeiro. Revista de Administração, v. 30, n. 1, p. 84-89. São Paulo. 1995.

AZZOLINI, B-H. et al. Diferença no preço de produtos convencionais e orgânicos e o perfil socioeconômico do consumidor de orgânico. Synergismus Scyentifica UTFPR, v. 2, (1, 2, 3, 4), Pato Branco. 2007.

BANKS, J., BLUNDELL, R. e LEWBEL, A. Quadratic Engel curves and consumer demand. The Review of Economics and Statistics, v. 79, n. 4, p. 527-539, nov. 1997.

BLUNDELL, R. e ROBIN, J. M. Estimation in large and disaggregated demand system: An estimator for conditionally linear systems. Journal of Applied Econometrics, v. 14, p. 209-232, 1999.

CERVEIRA, R. e CASTRO, M. C. Consumidores de produtos orgânicos da cidade de São Paulo: características de um padrão de consumo. Informações Econômicas, v. 29, n. 12. São Paulo. 1999.

COX, T. e WOHLGENANT, M. Prices and quality effects in cross-section demand analysis. The American Journal of Agricultural Economics, v. 68, n. 4, p. 908-919, 1986.

DEATON, A. The Analysis of household surveys. A Microeconometric Approach to Development Policy. Baltmore: Johns Hopkins University Press, 1997.

DHAR, T. e FOLTZ, J. D. 'Milk by any other name ... Consumer benefits from labeled milk'. American Journal of Agricultural Economics, v. 87, p. 214-218, 2005.

FOURMOUZI, V., GENIUS, M. e MIDMORE, P. The demand for organic and conventional produce in London, UK: a system approach. Journal of Agricultural Economics, v. 63, n. 3, p. 677-693, 2012.

GLASER, L. e THOMPSON, G. D. Demand for organic and conventional frozen vegetables. Annual Meeting of the American Agricultural Economics Association. Nashville, out. 1998.

Demand for organic and conventional beverage milk. Annual Meeting of the Western Agricultural Economics Association. Vancouver, june, 2000.

GRACIA, A. e MAGISTRIS, T. The demand for organic foods in the south of Italy: a discrete choice model. Food Policy, v. 33, p. 386-396, 2008.
IBGE - Instituto Brasileiro de Geografia e Estatítica. Microdados da POF (Pesquisa de Orçamentos Familiares). CD-Rom. Rio de Janeiro: 2010a.

. Pesquisa de Orçamentos Familiares: despesas, rendimentos e condições de vida. Rio de Janeiro: 2010b.

- Pesquisa de Orçamentos Familiares 2008-2009: aquisição alimentar domiciliar per capita Brasil e Grandes regiões. Rio de Janeiro: 2010c.

KASTERIDIS, P. e YEN, S. T. U.S. demand for organic and conventional vegetables: a Bayesian censored system approach. The Australian Journal of Agricultural and Resource Economics, v. 56, p. 405-425. 2012.

LI, J., ZEPEDA, L. e GOULD, B. W. The demand for organic food in the U.S.: an empirical assessment. Journal of food Research, v. 38, n. 3, 2007.

LIN, B-H. et al. U.S. demand for organic and conventional fresh fruits: the roles of income and price. Sustainability, v. 1, p. 464-478, 2009.

MAPA - Ministério da Agricultura, Pecuária e Abastecimento. Brasília. 2014. Disponível em: $<$ http://www.agricultura.gov.br/comunicacao/ noticias/2011/02/mercado-interno-de-organicoscresce-40porcento-em-2010 >. Acesso em: abr. 2014.

. Brasília. 2015. Cadastro Nacional de Produtores Orgânicos. Disponível em: <http://www.agricultura. gov.br/comunicacao/noticias/2015/03/numero-deprodutores-organicos-cresce-51 porcento-em-umano >. Acesso em: mar. 2015.

MAZOYER, M. e ROUDART, L. História das agriculturas no mundo: do neolítico à crise contemporânea. São Paulo. 2010. p. 500-501.

OLIVEIRA, F. C. R. e HOFFMANN, R. Consumo de alimentos orgânicos e de produtos light e diet no Brasil: fatores condicionantes e elasticidades-renda. Segurança Alimentar e Nutricional, v. 22, n. 1, p. 541-557, 2015.

ORGANICS BRASIL. Organics Brasil Imprensa. Disponível em: <http://www.organicsbrasil.org/ pt/imprensa_detalhe/173/projeto-organics-brasil $>$. Acesso em: abr. 2014.

POI, B. P. Demand-system estimation: update. The Stata Journal, v. 8, n. 4, p. 554-556, 2008.

PORTAL ORGÂNICO. Disponível em: < http://www. portalorganico.com.br/noticia/209/mercado_brasileiro_ de_organicos_deve_crescer_35_em_2014> . Acesso em: mar. 2015.

SCHRÖCK, R. Determinants of the demand for organic and conventional fresh milk in Germany - an 
econometric analysis. $1^{\circ}$ Joint EAAE/AAEA Seminar. Freising, Alemanha, set. 2010.

SHONKWILER, J. e YEN, S. Two-step estimation of a censored system of equations. American Journal of Agricultural Economics, v. 81, n. 4, p. 972-982, nov. 1999.

SMITH, T. A., HUANG C. L. e LIN, B-H. Does price or income affect organic choice? Analysis of U.S. fresh produce users. Journal of Agricultural and Applied Economics, v. 41, n. 3, p. 731-744, 2009.

SOUZA, M. C. M. Aspectos institucionais do sistema agroindustrial de produtos orgânicos. Informações Econômicas, v. 33, n. 3, p. 7-16, 2003.

TAFERE, K. et al. Food demand elasticities in Ethiopia: estimates using Household Income Consumption Expenditure (HICE) Survey Data. ESSP II, Adis Ababa: IFPRI/EDRI, 2010 (Working Paper n. 11)

TREGEAR, A., DENT, J. B. e MCGREGOR, M. J. The demand for organically- grow produce. British Food Journal, v. 96, n. 4, 1994.
WANG, Q. e SUN J. Consumer preference and demand for organic food: evidence from a Vermont survey. American Agricultural Economics Association Annual Meeting Montreal, Montreal, Canadá, 2003.

WILLER, H. e LERNOUD, J. (Eds.) (2014). The world of organic agriculture. Statistics and emergent trends 2014. Research Institute of Organic Agriculture (FiBL), Frick, e International Federation of Organic Agriculture Movements (IFOAM), Bonn. 2014.

YEN, S. T. e HUANG, C. L. Cross-sectional estimation of U.S. demand for beef products: a censored system approach. Journal of Agricultural and Ressource Economics, v. 27, n. 2 , p. 320-334, 2002.

., LIN, B. e SMALLWOOD, D. M. Quasi- and simulated-likelihood approaches to censored demand systems: food consumption by food stamp recipients in the United States, American Journal of Agricultural Economics, v. 85, p. 458-478, 2003. 
